\section{COVID-19 e (in)segurança alimentar e nutricional: ações do Governo Federal brasileiro na pandemia frente aos desmontes orçamentários e institucionais}

\author{
COVID-19 and food and nutritional (in)security: \\ action by the Brazilian Federal Government \\ during the pandemic, with budget cuts and \\ institutional dismantlement
}

\author{
COVID-19 e (in)seguridad alimentaria y \\ nutricional: acciones del Gobierno Federal \\ brasileño en la pandemia frente a los recortes \\ presupuestarios e institucionales
}

Tais de Moura Ariza Alpino 1 Cláudia Roberta Bocca Santos 2 Denise Cavalcante de Barros 3 Carlos Machado de Freitas 1

doi: 10.1590/0102-311X00161320

\section{Resumo}

A pandemia por COVID-19 representa um dos maiores desafios da saúde pública deste século, causando impactos na saúde e nas condições de vida das populações em todo o mundo. Tem sido apontado pela literatura que a pandemia afeta de diversas formas o sistema alimentar hegemônico. No Brasil, a pandemia amplifica as desigualdades sociais, raciais e de gênero já existentes, comprometendo ainda mais a garantia do Direito Humano à Alimentação Adequada (DHAA) e a concretização da segurança alimentar e nutricional, especialmente entre os mais vulneráveis. Nesse contexto, este artigo tem como objetivo analisar as primeiras ações, em âmbito federal, do governo brasileiro para a mitigação dos efeitos da pandemia que podem repercutir na segurança alimentar e nutricional, considerando as recentes mudanças institucionais das politicas e programas. Foi realizada uma revisão narrativa da literatura e utilizado como fontes de informação os boletins do Centro de Coordenação de Operações do Comitê de Crise para Supervisão e Monitoramento dos Impactos da COVID-19 e homepages de ministérios setoriais, de março a maio de 2020. As ações foram sistematizadas segundo as diretrizes da Política Nacional de Segurança Alimentar e Nutricional. Foi identificada a criação de arranjos institucionais para o gerenciamento da crise. Dentre as ações propostas, destacam-se aquelas relacionadas ao acesso à renda, como o auxílio emergencial, e a alimentos, como a autorização para a distribuição de alimentos fora do ambiente escolar com os recursos federais do Programa Nacional de Alimentação Escolar. No entanto, os retrocessos e desmontes na área de segurança alimentar e nutricional podem comprometer a capacidade de resposta do Governo Federal no contexto da COVID-19.

Segurança Alimentar e Nutricional; COVID-19; Política Pública

\author{
Correspondência \\ T. M. A. Alpino \\ Centro de Estudos e Pesquisas em Emergências e Desastres em \\ Saúde, Fundação Oswaldo Cruz. \\ Av. Brasil 4036, sala 916, Rio de Janeiro, RJ 21040-361, Brasil. \\ arizatais@gmail.com \\ 1 Centro de Estudos e Pesquisas em Emergências e Desastres em \\ Saúde, Fundação Oswaldo Cruz, Rio de Janeiro, Brasil. \\ 2 Escola de Nutrição, Universidade Federal do Estado do Rio de \\ Janeiro, Rio de Janeiro, Brasil. \\ 3 Escola Nacional de Saúde Pública Sergio Arouca, Fundação \\ Oswaldo Cruz, Rio de Janeiro, Brasil.
}




\section{Introdução}

A pandemia por COVID-19 representa um dos maiores desafios sanitários mundiais deste século, causando impactos diretos e indiretos 1,2. No Brasil, o primeiro caso foi confirmado em fevereiro e até 1o de julho foram 1.448.753 casos e 60.632 mortes (Ministério da Saúde. Painel Coronavírus. https://covid.saude.gov.br/, acessado em 01/Jul/2020). O insuficiente conhecimento científico sobre a doença associado à sua acelerada expansão geram incertezas quanto à definição das estratégias para o seu enfrentamento.

No Brasil, uma das principais medidas foi o isolamento social, sendo alvo de debates em função das repercussões econômicas, sociais e psicológicas 2,3, e colocado em prática em distintos graus entre os estados e municípios brasileiros. A postura cética de autoridades, como a do Presidente da República, que não acredita na eficácia dessa medida no controle da pandemia 4,5 com um discurso divergente dos ministros da saúde 6 , além da falta da adoção de ações convergentes com critérios comuns pelos entes federados em relação ao isolamento social 7 , a troca de dois ministros da saúde e a ausência há mais de 40 dias de um ministro efetivo ${ }^{8}$ podem afetar a eficácia dessa medida de combate à pandemia e a adesão da população.

Outro ponto a ser discutido é que mesmo o isolamento sendo uma medida necessária, este não pode estar submisso à lógica econômica, devendo estar atrelado à garantia dos direitos humanos 2,9. Nesse sentido, os desafios são maiores num país como o Brasil, pois pouco se sabe sobre as características de transmissão num contexto de desigualdade social e demográfica, com condições precárias de habitação e saneamento, sem acesso regular à água, em situação de aglomeração, alta prevalência de doenças crônicas, com inúmeras violações de direitos humanos 2. Entre eles, está o Direito Humano à Alimentação Adequada (DHAA) e a concretização da segurança alimentar e nutricional.

Sabe-se que a segurança alimentar e nutricional pode ser afetada pelos impactos sociais e econômicos da COVID-19, especialmente se considerarmos as situações de desigualdade social, de renda, étnico-racial, de gênero e de acesso a serviços de saúde 10. O Relatório Global sobre Crises Alimentares 11 estima que 135 milhões de pessoas apresentavam insegurança alimentar em 2019, mas em entrevista o economista Daniel Balaban, do Programa Mundial de Alimentos, projeta que devido aos efeitos econômicos e interrupções da cadeia de suprimentos decorrentes da COVID-19 este número poderia alcançar 265 milhões de pessoas 12. A pandemia afeta a oferta e demanda de alimentos, reduz o poder de compra e a capacidade de produzir e distribuir alimentos, afetando especialmente os mais vulneráveis.

Dessa forma, o papel do Estado é crucial para mitigar os efeitos da pandemia, com medidas de curto, médio e longo prazos, não apenas para o controle da COVID-19, como também das consequências. Este texto pretende analisar as primeiras ações, em âmbito federal, do governo brasileiro para a mitigação dos efeitos da pandemia que podem repercutir na segurança alimentar e nutricional, considerando as recentes mudanças institucionais das políticas e programas.

\section{Procedimentos metodológicos}

Trata-se de estudo empírico com análise de fontes bibliográficas e documentais. Para identificar as ações do Governo Federal para a mitigação dos efeitos da pandemia que podem ter efeitos na segurança alimentar e nutricional, foram analisados os boletins do Centro de Coordenação de Operações do Comitê de Crise para Supervisão e Monitoramento dos Impactos da COVID-19 (CCOP) no site da Casa Civil (https://www.gov.br/casacivil/pt-br), além das homepages de ministérios setoriais, no período de março a maio de 2020. Em seguida, as ações foram sistematizadas segundo as diretrizes da Política Nacional de Segurança Alimentar e Nutricional (PNSAN). Todas as ações identificadas no período citado que poderiam ter repercussões na segurança alimentar e nutricional foram incluídas no presente estudo.

Foi também realizada uma revisão narrativa da literatura, utilizando artigos científicos e posicionamentos de entidades da sociedade civil organizada sobre as mudanças recentes institucionais nas políticas públicas e programas de segurança alimentar e nutricional no país, além de documen- 
tos nacionais e internacionais com foco na relação COVID-19 e segurança alimentar e nutricional 10,13,14,15, adotados como referencial teórico para a análise das ações do Governo Federal durante a pandemia. Revisões narrativas buscam descrever e discutir o "estado da arte" sobre um assunto, sob o ponto de vista teórico ou contextual. Esse tipo de revisão não apresenta reprodução metodológica, porém contribui com questões pertinentes e colabora para a atualização do conhecimento sobre um tema em curto espaço de tempo 16.

O processo de coleta dos artigos e documentos foi realizado de forma não sistemática no período de março a maio de 2020. Foram pesquisadas bases de dados científicas e homepages institucionais, como Google Acadêmico; SciELO; Organização das Nações Unidas para a Alimentação e a Agricultura (FAO); Comitê de Segurança Alimentar Mundial (CFS); FIAN Internacional e Brasil; e Fórum Brasileiro de Soberania e Segurança Alimentar (FBSSAN). Além dessas fontes, foram usadas reportagens jornalísticas a fim de identificar atualidades sobre o tema em questão.

\section{Resultados e discussão}

A pandemia está em evolução e pouco se sabe sobre sua dinâmica, duração e alcance geográfico, sendo difícil mensurar seus impactos. Mas é reconhecido que medidas de isolamento social poderão impactar no acesso à renda e afetar a curto, médio e longo prazos a regularidade da produção, acesso, disponibilidade, abastecimento e preço de alimentos e, consequentemente, a garantia da segurança alimentar e nutricional e do DHAA 17,18,19,20. As formas pelas quais os países, governos e instituições respondem politicamente à pandemia podem ter profundas implicações na garantia do DHAA. Respostas políticas por meio de ações sem articulação com diversos setores do governo e pouco orientadas por dados científicos podem aumentar os impactos sociais, econômicos e de saúde na pandemia 19.

No Brasil, as ações do Governo Federal frente à pandemia no período analisado envolveram a criação de arranjos institucionais, disponíveis no Quadro 1.

Uma das primeiras medidas foi a instituição do Grupo Executivo Interministerial de Emergência em Saúde Pública de Importância Nacional e Internacional (GEI-ESPII), datada de 30 de janeiro de 2020, com o objetivo de propor, acompanhar e articular medidas de preparação e de enfrentamento às emergências em saúde pública 21,22. Poucos dias depois, o Brasil declarou Emergência em Saúde Pública de Importância Nacional 23. Mais de um mês após, o Comitê de Crise foi instituído 24. Além dos arranjos institucionais, cabe destacar as ações iniciais para a mitigação dos efeitos da COVID-19, que podem afetar a segurança alimentar e nutricional (Quadro 2).

Algumas ações sistematizadas no Quadro 2 serão discutidas ao longo desta seção.

\section{Promoção do acesso universal à alimentação adequada saudável, com prioridade para as famílias e pessoas em situação de insegurança alimentar e nutricional}

O auxílio emergencial estabelece medidas excepcionais de proteção social para trabalhadores informais, microempreendedores individuais, autônomos e desempregados 25. Foi proposto o valor de R\$ 200,00, porém, após articulações envolvendo grupos políticos e votação na Câmara dos Deputados, o valor foi triplicado, por três meses 26 . Em 30 de junho de 2020, o Presidente Jair Bolsonaro, por meio do Decreto no 10.412, prorrogou por mais dois meses o auxílio 27. Beneficiários do Programa Bolsa Família podem receber o auxílio, desde que seja mais vantajoso e nesse período o Programa Bolsa Família ficará suspenso, a despeito de focalizar famílias em maior vulnerabilidade social.

Em todo o mundo, os países têm adotado transferência direta de renda como ação contra a crise econômica 28 , que tem implicações enormes para a segurança alimentar e nutricional, a exemplo das pessoas que trabalham como mão de obra informal, serviços, restaurantes e varejo. Esses trabalhadores podem enfrentar perda de empregos, seja pelo distanciamento social ou pela desaceleração econômica. Embora os produtores de alimentos tenham demanda para a produção, as interrupções nas cadeias alimentares e nos mercados agroalimentares podem alterar os meios de subsistência dos agricultores familiares, assim como a produção e disponibilidade de alimentos. Essas situações têm implicações diretas no acesso das pessoas à alimentação e à água 15. 


\section{Quadro 1}

Arranjos institucionais do Governo Federal para mitigação dos efeitos da pandemia que podem afetar a segurança alimentar e nutricional no período de janeiro a maio de 2020 .

\begin{tabular}{|c|c|}
\hline $\begin{array}{l}\text { Arranjos institucionais do Governo Federal para mitigação dos } \\
\text { efeitos da COVID-19 }\end{array}$ & Objetivos e composição \\
\hline $\begin{array}{l}\text { Grupo Executivo Interministerial de Emergência em Saúde Pública } \\
\text { de Importância Nacional e Internacional (GEI-ESPII). } \\
\text { Decreto no } 10.211 \text {, de } 30 \text { de janeiro de } 202021 \text { e Decreto no 10.238, } \\
\text { de } 11 \text { de fevereiro de } 202022 \text {. }\end{array}$ & $\begin{array}{l}\text { Instituído para propor, acompanhar e articular medidas de preparação } \\
\text { e de enfrentamento às emergências em saúde pública. Sua composição } \\
\text { foi alterada por Decreto posterior, sendo composto pelo Ministério da } \\
\text { Saúde, que o coordena; Casa Civil da Presidência da República, além de } \\
\text { outros Ministérios e órgãos da administração pública. }\end{array}$ \\
\hline $\begin{array}{l}\text { Comitê de Crise para Supervisão e Monitoramento dos Impactos } \\
\text { da COVID-19. } \\
\text { Decreto no } 10.277 \text {, de } 16 \text { de março de } 202024 \text {. }\end{array}$ & $\begin{array}{l}\text { Coordenado pelo Ministro de Estado Chefe da Casa Civil, constitui um } \\
\text { órgão de articulação da ação governamental e de assessoramento ao } \\
\text { Presidente, sendo formado por dirigentes de ministérios e órgãos da } \\
\text { administração pública, além do Coordenador do Centro de Operações de } \\
\text { Emergências em Saúde Pública da Secretaria de Vigilância em Saúde do } \\
\text { Ministério da Saúde, devendo ter atuação coordenada com o GEI-ESPII. }\end{array}$ \\
\hline $\begin{array}{l}\text { Comitê de Crise (CC-AGRO-COVID19). } \\
\text { Portaria no 123, de } 30 \text { de março de } 202081 .\end{array}$ & $\begin{array}{l}\text { No âmbito do Ministério da Agricultura, Pecuária e Abastecimento } \\
\text { foi instituído Comitê de Crise com a finalidade de monitorar e propor } \\
\text { estratégias para minimizar os impactos do coronavírus na produção } \\
\text { agrícola e no abastecimento de alimentos. Esta Portaria estabelece } \\
\text { que compete ao CC-AGRO-COVID19 “analisar produção, mercado, } \\
\text { infraestrutura, percepções da sociedade e produtos agropecuários; analisar } \\
\text { cenários e produzir diagnósticos para subsidiar a gestão do Ministério da } \\
\text { Agricultura, Pecuária e Abastecimento; analisar e acompanhar questões } \\
\text { com potencial de risco, prevenir a ocorrência de crises e articular seu } \\
\text { gerenciamento, em caso de grave e iminente ameaça ao abastecimento de } \\
\text { produtos e insumos agropecuários; e propor ações que contribuam para a } \\
\text { manutenção do abastecimento da população brasileira". }\end{array}$ \\
\hline $\begin{array}{l}\text { Conselho de Solidariedade para Combate à COVID-19 e aos seus } \\
\text { efeitos sociais e econômicos. } \\
\text { Decreto no } 10.311 \text {, de } 3 \text { de abril de } 202082 \text {. }\end{array}$ & $\begin{array}{l}\text { Este conselho visa a orientar a execução de ações financiadas por } \\
\text { doações destinadas ao enfrentamento da COVID-19. É constituído por } \\
\text { representantes da Casa Civil, de Ministérios e da Secretaria de Governo } \\
\text { da Presidência da República. A primeira ação foi a definição para a } \\
\text { utilização dos recursos do projeto Arrecadação Solidária, pelo qual } \\
\text { pessoas físicas e jurídicas podem realizar doações financeiras. }\end{array}$ \\
\hline
\end{tabular}

Fonte: elaboração própria. 


\section{Quadro 2}

Ações do Governo Federal para mitigação dos efeitos da pandemia que podem afetar a segurança alimentar e nutricional no período de março a maio de 2020 .

\begin{tabular}{|c|c|}
\hline $\begin{array}{l}\text { Diretrizes da Política } \\
\text { Nacional de Segurança } \\
\text { Alimentar e Nutricional } \\
\text { (PNSAN) }\end{array}$ & Ações identificadas \\
\hline \multirow{6}{*}{$\begin{array}{l}\text { Promoção do acesso universal } \\
\text { à alimentação adequada e } \\
\text { saudável, com prioridade } \\
\text { para as famílias e pessoas } \\
\text { em situação de insegurança } \\
\text { alimentar e nutricional. }\end{array}$} & Instituição da Renda Básica Emergencial (Lei no 13.982, de 2 de abril de 2020 25). \\
\hline & Antecipação do auxílio emergencial a pessoas que requereram o Benefício de Prestação Continuada (BPC). \\
\hline & $\begin{array}{l}\text { Adequação das normas do Programa Nacional de Alimentação Escolar (PNAE) para a continuidade do programa } \\
\text { durante o período de pandemia (Lei no 13.987, de } 7 \text { de abril de } 2020 \text { 43) e antecipação de repasse financeiro do } \\
\text { Fundo Nacional de Desenvolvimento da Educação (FNDE) para o PNAE aos estados e municípios. }\end{array}$ \\
\hline & $\begin{array}{l}\text { Aporte financeiro para pequenas e médias empresas brasileiras, a fim de garantir a manutenção } \\
\text { dos salários dos trabalhadores. }\end{array}$ \\
\hline & $\begin{array}{l}\text { Repasse emergencial de recursos federais para a operacionalização de ações de incremento à segurança } \\
\text { alimentar e nutricional aos usuários do Sistema Único de Assistência Social - SUAS } \\
\text { (Portaria no 385, de } 13 \text { de maio de } 2020 \text { 49). }\end{array}$ \\
\hline & $\begin{array}{l}\text { Acréscimo de medidas emergenciais na gestão do Programa Bolsa Família } \\
\qquad(\text { Portaria no 387, de } 15 \text { de maio de } 2020 \text { 36). }\end{array}$ \\
\hline \multirow{15}{*}{$\begin{array}{l}\text { Promoção do abastecimento } \\
\text { e estruturação de } \\
\text { sistemas sustentáveis e } \\
\text { descentralizados, de base } \\
\text { agroecológica, de produção, } \\
\text { extração, processamento e } \\
\text { distribuição de alimentos. }\end{array}$} & $\begin{array}{l}\text { Criação de canal de WhatsApp para a comunicação de perdas na agricultura em função da COVID-19 } \\
\text { (Disque Perdas de Alimentos). }\end{array}$ \\
\hline & $\begin{array}{l}\text { Instituição da Portaria no 116, de } 26 \text { de março de } 2020 \text { 83, que dispõe sobre os serviços, as atividades e os } \\
\text { produtos considerados essenciais pelo Ministério da Agricultura, Pecuária e Abastecimento para } \\
\text { o pleno funcionamento das cadeias produtivas de alimentos e bebidas, para assegurar o } \\
\text { abastecimento e a segurança alimentar da população brasileira enquanto perdurar o estado } \\
\text { de calamidade pública decorrente da pandemia da COVID-19. }\end{array}$ \\
\hline & Elaboração de recomendações de boas práticas em feiras livres. \\
\hline & Publicação de orientações para a segurança do trabalhador em ambiente frigorífico. \\
\hline & $\begin{array}{l}\text { Publicação da Resolução no } 4.80157 \text { que autoriza, para produtores rurais, inclusive agricultores familiares, a } \\
\text { prorrogação do reembolso das operações de crédito rural; a contratação de Financiamento para Garantia } \\
\text { de Preços ao Produtor (FGPP) com recursos do crédito rural para estocagem e comercialização; e cria linhas } \\
\text { especiais de crédito de custeio ao amparo do Programa Nacional de Fortalecimento da Agricultura Familiar } \\
\text { (Pronaf) e do Programa Nacional de Apoio ao Médio Produtor Rural (Pronamp) com taxas de juros subsidiadas. }\end{array}$ \\
\hline & $\begin{array}{l}\text { Monitoramento dos principais estabelecimentos de abastecimento (Companhia Nacional de Abastecimento, } \\
\text { centrais de abastecimentos e supermercados). }\end{array}$ \\
\hline & $\begin{array}{l}\text { Elaboração de recomendações para os setores relacionados ao abastecimento alimentar } \\
\text { (colheita, transporte e comercialização). }\end{array}$ \\
\hline & $\begin{array}{l}\text { Abertura de crédito extraordinário de R\$500 milhões para a compra de produtos da agricultura familiar por } \\
\text { meio do Programa de Aquisição de Alimentos - PAA (Medida Provisória no 957, de } 24 \text { de abril de 2020 53). }\end{array}$ \\
\hline & $\begin{array}{l}\text { Prorrogação da validade de Declarações de Aptidão ao Pronaf (DAPs) por seis meses pelo Ministério da } \\
\text { Agricultura, Pecuária e Abastecimento (Portaria no 24, de } 24 \text { de março de } 2020 \text { 84). }\end{array}$ \\
\hline & $\begin{array}{l}\text { Criação de canal direto on-line no portal (http://www.gov.br) junto ao Ministério da Agricultura, Pecuária e } \\
\text { Abastecimento para representações do setor agropecuário visando a receber pedidos } \\
\text { de ação em decorrência da pandemia. }\end{array}$ \\
\hline & Contratação de crédito rural em oito meses para produtores. \\
\hline & Liberação de linhas de crédito de para pequenos empreendedores, cooperativas e informais. \\
\hline & $\begin{array}{r}\text { Publicação de Instrução Normativa no 29, de } 23 \text { de abril de } 2020 \text { 59, que estabelece requisitos para o livre comércio } \\
\text { de produtos de origem animal, inspecionados por consórcio público de municípios. }\end{array}$ \\
\hline & $\begin{array}{l}\text { Definição de medidas de caráter emergencial para os procedimentos relativos à concessão, ao controle e à } \\
\text { fiscalização das operações de crédito rural (Resolução no 4.810, de } 30 \text { de abril de } 2020 \text { 57). }\end{array}$ \\
\hline & $\begin{array}{l}\text { Mudança de regras do Garantia-Safra para a verificação das perdas por seca nos municípios pelo Ministério da } \\
\text { Agricultura, Pecuária e Abastecimento e antecipação do pagamento do Garantia-Safra. }\end{array}$ \\
\hline
\end{tabular}

(continua) 
Quadro 2 (continuação)

\section{Diretrizes da Política Nacional de Segurança Alimentar e Nutricional (PNSAN)}

Instituição de processos

permanentes de educação alimentar e nutricional, pesquisa e formação nas áreas de segurança alimentar e nutricional e do direito humano à alimentação adequada.

Promoção, universalização e coordenação das ações de segurança alimentar e nutricional voltadas para quilombolas e demais povos e comunidades tradicionais de que trata o art. 3o, inciso I, do Decreto no 6.040, de 7 de fevereiro de 200785 , povos indígenas e assentados da reforma agrária.

Fortalecimento das ações de alimentação e nutrição em todos os níveis da atenção à saúde, de modo articulado às demais ações de segurança alimentar e nutricional.

Promoção do acesso universal à água de qualidade e em quantidade suficiente, com prioridade para as famílias em situação de insegurança hídrica e para a produção de alimentos da agricultura familiar e da pesca e aquicultura.
Ações identificadas

Publicação de materiais educativos da Secretaria de Vigilância em Saúde e da Secretaria de Atenção Primária à Saúde no contexto da pandemia com recomendações sobre alimentação, como: planejamento de refeições e de compras de alimentos, cuidados e higienização de hortaliças e vegetais, aleitamento materno, alimentação de idosos.

Doação de cestas básicas e kits alimentação para grupos populacionais específicos.

Elaboração de recomendações da Coordenação Geral de Alimentação e Nutrição (CGAN) às Secretarias Estaduais de Saúde e do Distrito Federal, visando a contribuir para a saúde e a segurança alimentar e nutricional no contexto da pandemia.

(continua) 


\begin{tabular}{|l|l|}
\hline Diretrizes da Política & Ações identificadas \\
Nacional de Segurança & \\
Alimentar e Nutricional & \\
(PNSAN) & Não foram identificadas ações. \\
\hline Apoio a iniciativas de & \\
promoção da soberania & \\
alimentar, segurança & \\
alimentar e nutricional e do & \\
direito humano à alimentação & \\
adequada em âmbito & \\
internacional e a negociações & \\
internacionais baseadas nos & \\
princípios e diretrizes da Lei no & \\
11.346, de 15 de setembro de & \\
2006 86. & \\
\hline Monitoramento da realização & \\
do Direito Humano à & Não foram identificadas ações. \\
Alimentação Adequada. & \\
\hline
\end{tabular}

Fonte: elaboração própria.

Nesse contexto, é preciso dar luz ao veto presidencial que rejeitou a ampliação para os trabalhadores que não estão incluídos no Cadastro Único para programas sociais do Governo Federal 29, o que amplifica a possibilidade de insegurança alimentar e nutricional e fome em grupos já historicamente alijados da perspectiva de direito. Por outro lado, o Governo Federal sancionou o pagamento do auxílio para mães menores de 18 anos 29,30,31. O veto aos agricultores familiares, de um lado, condena parte dessa população a situações precárias de vida e, de outro, põe em risco o abastecimento alimentar 32.

O auxílio emergencial foi popularmente nomeado como coronavoucher, o que reflete um caráter pejorativo, como algo perecível, estigmatizante, e não a perspectiva do direito e dever do Estado para além de uma renda emergencial para os mais vulneráveis ${ }^{33}$. Diariamente, são noticiados problemas no processo de garantia desse direito que geraram aglomerações nas agências da Caixa Econômica Federal, contrapondo as recomendações da Organização Mundial da Saúde (OMS) 34,35. Dessa forma, a pandemia escancara e amplifica as desigualdades existentes no Brasil e a necessidade de efetivar políticas universais de renda básica.

Também foi identificada a antecipação do auxílio emergencial a pessoas que requereram o Benefício de Prestação Continuada (BPC) e alterações na gestão do Programa Bolsa Família, com o objetivo de evitar aglomerações para alterações e inclusões de cadastros 36 . A expansão dos programas é sugerida no documento do FBSSAN 10, a fim de aumentar a renda das famílias e possibilitar o acesso à alimentação. Segundo Burlandy 37, programas de transferência de renda podem impactar o bem-estar nutricional dos beneficiários, assim como proporcionar a compra de bens não alimentares, como de equipamentos domésticos que possibilitem armazenar ou processar alimentos. Apesar dos efeitos positivos do Programa Bolsa Família 38,39, o foco da atual gestão tem sido a busca por irregularidades e cancelamento de benefícios, com base no cruzamento de informações de bancos de dados 40,41. Em 2020, o Programa Bolsa Família tem verba prevista de R\$ 29,5 bilhões, abaixo dos R \$ 32 bilhões direcionados ao programa em 2019 42. Tais medidas afetam diretamente as populações mais vulneráveis do país no que diz respeito ao acesso à renda, e podem exacerbar os efeitos da pandemia na segurança alimentar e nutricional.

O desenho do Programa Nacional de Alimentação Escolar (PNAE) não previa a excepcionalidade de uma emergência sanitária. Com o fechamento das escolas, os estudantes ficaram desassistidos e a 
compra dos gêneros alimentícios da agricultura familiar foi prejudicada. Sendo assim, foi instituída a Lei no 13.987, que prevê que o Fundo Nacional de Desenvolvimento da Educação (FNDE) deve manter a transferência de recursos aos estados e municípios, autorizando sua destinação para a compra e distribuição de gêneros aos pais ou responsáveis dos estudantes, mantendo a exigência de compra da agricultura familiar 43,44. Os recursos federais não podem ser destinados à transferência direta de renda às famílias, tampouco apoiar custos de distribuição de alimentos.

É importante sinalizar que estão ocorrendo descompassos quanto à implementação da normativa nos contextos locais, e alguns entes federados sequer estão implementando estratégias para a garantia deste direito 45,46. Considerando que a compra de gêneros da agricultura familiar para o PNAE representa uma convergência virtuosa de políticas públicas, os gêneros ofertados às famílias devem respeitar essa normativa ${ }^{47}$. Valadares et al. ${ }^{47}$ sugerem que, diante da necessidade das entidades executoras adequarem a logística de distribuição de alimentos, o FNDE deveria considerar o aumento dos recursos, visto que o aumento do custo da execução do programa, considerando as adequações necessárias durante a pandemia, pode comprometer a continuidade do mesmo num contexto de restrição orçamentária. Os documentos utilizados como referencial teórico por este manuscrito reforçam a necessidade de continuidade do PNAE, ressaltando o papel do programa na garantia do DHAA, especialmente na pandemia 10,13,14.

Foi autorizado um recurso emergencial do Governo Federal com a finalidade de aumentar a capacidade de resposta do Sistema Único de Assistência Social (SUAS) no atendimento às famílias e aos indivíduos em situação de vulnerabilidade e risco social decorrente da COVID-19, promovendo a compra de alimentos, prioritariamente ricos em proteína, para pessoas idosas e com deficiências acolhidas no Serviço de Acolhimento Institucional e em atendimento no Serviço de Proteção Social Especial para Pessoas com Deficiência, Idosas e suas Famílias 48. Outro repasse emergencial de recursos federais também foi autorizado para a operacionalização de ações de incremento à segurança alimentar e nutricional aos usuários do SUAS 49. Apesar da falta de clareza sobre a utilização desse recurso, o Ministério da Cidadania aponta que atenderá famílias e indivíduos em vulnerabilidade. Santarelli et al. 40 apontam que, desde o governo do Presidente Michel Temer e intensificado com Jair Bolsonaro, ocorre uma crescente e continuada redução dos programas de segurança alimentar e nutricional. A rede de equipamentos públicos de segurança alimentar e nutricional deixou de ser uma prioridade para o Governo Federal, que não realiza editais para a descentralização de recursos, e para os estados e municípios, que fecharam unidades. Houve também redução orçamentária do programa de cestas de alimentos.

\section{Promoção do abastecimento e estruturação de sistemas sustentáveis e descentralizados, de base agroecológica, de produção, extração, processamento e distribuição de alimentos}

Destaca-se, dentre as Medidas Provisórias publicadas 50,51,52,53, a de no 957 53, que abre crédito extraordinário de R $\$ 500$ milhões para a compra de produtos da agricultura familiar pelo Programa de Aquisição de Alimentos (PAA). Segundo o Ministério da Cidadania, 85 mil famílias de agricultores familiares serão beneficiadas, além de 12,5 mil entidades e 11 milhões de famílias em vulnerabilidade social, que receberão os alimentos 54 . A abertura de crédito em favor do PAA acontece após a mobilização da sociedade civil organizada, que apontou a necessidade de recursos imediatos de R\$ 1 bilhão para o Programa 55.

É importante destacar que a pandemia joga luz nos desmontes orçamentários e institucionais em programas de segurança alimentar e nutricional. Por exemplo, o desmonte do Ministério do Desenvolvimento Agrário no Governo Temer, seguido da extinção da Secretaria Nacional de Segurança Alimentar e Nutricional (SESAN) com Bolsonaro, que era uma instância estratégica na coordenação intersetorial da PNSAN e na descentralização do Sistema Nacional de Segurança Alimentar e Nutricional (SISAN), bem como mudanças na gestão e cortes orçamentários de programas centrais, caso do PAA 40.

Para Valadares et al. 47 (p. 7), diante dos cortes orçamentários realizados, "torna-se praticamente inviável para os pequenos produtores contar apenas com uma saída 'de mercado' para sobreviver economicamen$t e^{\prime \prime}$ o que pode provocar perdas de produção, endividamento dos agricultores e afetar o abastecimento 
alimentar. Os autores apontam ainda que a retomada do PAA deveria privilegiar a modalidade de compra com doação de alimentos, associada à distribuição de cestas básicas e ao abastecimento da rede socioassistencial. Nesse sentido, ainda que tenha ocorrido repasse financeiro ao Programa, fica a preocupação se esta medida será suficiente dado o desmonte do PAA e da própria Companhia Nacional de Abastecimento (CONAB), visto que durante o Governo Bolsonaro houve um desmonte dos estoques públicos 56. Segundo Menezes \& Burity 32, as medidas no PNAE e no PAA não conseguiram contemplar os produtores que deveriam ser beneficiados.

A Resolução no 4.810 define medidas emergenciais para os procedimentos relativos ao crédito rural 57 . Maluf 17 sinaliza a necessidade da criação de linha de crédito emergencial para agricultores, com taxas de juros subsidiadas. O fortalecimento e a promoção da agricultura familiar são considerados essenciais para garantir que os pequenos produtores de alimentos tenham redes de apoio para manter sua capacidade de produzir e fornecer alimentos 10,13,14.

O setor do agronegócio continua crescendo, sendo considerado fundamental pelo governo para a recuperação econômica 58 . Nesse contexto, duas notícias identificadas nos boletins chamam atenção. A primeira é uma videoconferência realizada pela ministra do Ministério da Agricultura, Pecuária e Abastecimento com adidos agrícolas, cargo relacionado à expansão do agronegócio e ao gerenciamento de crises, buscando vender uma imagem positiva do Brasil como fornecedor de alimentos no exterior, o que demonstra a preocupação e foco no impacto da crise no agronegócio brasileiro. A segunda notícia aponta a preocupação com a queda de preços de carne bovina entre fevereiro e março, seguida da Instrução Normativa no 2959 que facilita o comércio de produtos de origem animal, inspecionados por consórcio público de municípios. É ainda importante destacar que o Governo Federal continua aprovando a liberação e a comercialização de novos agrotóxicos: em maio, mais 22 substâncias foram liberadas, totalizando 150 novas autorizações este ano 60 . Até poucos anos o Brasil era referência internacional para políticas referentes à agricultura familiar e atualmente tem um modelo de desenvolvimento rural com a liberação recorde de agrotóxicos, que oferece sérias ameaças à soberania alimentar 40,41,61,62.

As mudanças nas regras do Garantia-Safra para a verificação das perdas por seca e a antecipação do pagamento deste programa foram identificadas 63 . Essas ações são específicas para o semiárido que sofre com secas frequentes, contribuindo para amenizar as consequências negativas desse desastre 64 . Essa ação por si só não muda o retrato da pobreza e desigualdade histórica do semiárido, devendo estar associada a outras políticas de garantia dos direitos humanos, inclusive de acesso à alimentação, água e terra.

\section{Instituição de processos permanentes de educação alimentar e nutricional, pesquisa e formação nas áreas de segurança alimentar e nutricional e do direito humano à alimentação adequada}

Foi identificado um documento organizado pela Coordenação Geral de Alimentação e Nutrição (CGAN) do Ministério da Saúde, com recomendações sobre alimentação e COVID-19 65. É importante salientar que a pandemia e o isolamento social atingem diferentemente os indivíduos, afetando distintos determinantes da segurança alimentar e nutricional. Uma questão que pode favorecer a insegurança alimentar e nutricional é o maior consumo de ultraprocessados 66,67, por exemplo, em função da dificuldade de acesso a alimentos, pela falta de habilidades culinárias ou ainda pela desigual divisão sexual do trabalho doméstico, afetando mulheres que, diante de um novo cenário de atribuições, podem se ver dependentes de tais produtos. Parte dessa agenda pode ser alvo de Educação Alimentar e Nutricional, visando a apoiar indivíduos no desenvolvimento de habilidades culinárias. Os documentos do CFS e do Painel Internacional de Especialistas em Sistemas Alimentares Sustentáveis (IPES-Food) apontam que a COVID-19 irá influenciar os padrões alimentares e as diversas formas de má nutrição. As pessoas consumirão mais itens ultraprocessados como resultado das interrupções na cadeia de suprimentos e do pânico na compra de alimentos, e poderão reduzir o consumo de frutas, legumes e verduras, menos disponíveis em cadeias convencionais 15,18. No Brasil, uma pesquisa da Embrapa aponta outro padrão sobre o comportamento do consumidor de hortaliças. Para a maioria dos entrevistados $(72,5 \%)$ as hortaliças continuam acessíveis para a compra como no período anterior à pandemia e ao isolamento social. No entanto, essa situação varia conforme a renda familiar e a 
região do país, sendo que aqueles com maior nível salarial têm maior acesso a hortaliças, assim como os consumidores das regiões Sul e Sudeste 68.

Promoção, universalização e coordenação das ações de segurança alimentar e nutricional voltadas para quilombolas e demais povos e comunidades tradicionais, povos indígenas e assentados da reforma agrária

A doação de cestas básicas tem sido adotada, especialmente, para comunidades como ciganos, indígenas, ribeirinhos e vila de pescadores. Também foi identificada a distribuição de alimentos e água para famílias em situação de vulnerabilidade. A despeito da falta de clareza das informações presentes nos boletins e homepages de ministérios setoriais, foram identificados avisos de compras públicas para alimentos como macarrão, leite em pó, feijão, farinha de trigo, açúcar, óleo de soja, farinha de mandioca, flocos de milho, fubá de milho e arroz, todos destinados ao Ministério da Mulher, da Família e dos Direitos Humanos, para a distribuição de cestas básicas a comunidades tradicionais, operacionalizada pela CONAB. Não foram identificadas menções à oferta de frutas, legumes e verduras, tampouco aqueles advindos da agricultura familiar.

\section{Lacunas nas ações do Governo Federal}

A sistematização das ações tendo como referência as diretrizes da PNSAN permitiu identificar lacunas. Não foram identificadas ações referentes ao direito à água, especialmente quanto ao Programa Um Milhão de Cisternas (P1MC), que sofreu uma drástica redução orçamentária desde o Governo Temer. A desarticulação e o enfraquecimento desse Programa, em decorrência das restrições orçamentárias e redução do número de cisternas, afetam o acesso à água para o consumo e para a produção agrícola 40,69. A garantia do acesso à água potável foi apontada como central pelos documentos analisados 10,14

Quanto à diretriz que prevê o diálogo com o Sistema Único de Saúde (SUS), foi identificada a divulgação do Ofício Circular no 370 , com recomendações da CGAN às Secretarias Estaduais de Saúde e do Distrito Federal, visando a contribuir para a saúde e a segurança alimentar e nutricional no contexto da pandemia. Medidas recentes podem comprometer a atenção nutricional: o novo modelo de financiamento da atenção básica ${ }^{71}$; a revogação das normativas que definiam os parâmetros e 0 custeio do Núcleo Ampliado de Saúde da Família e o não credenciamento, pelo Ministério da Saúde, de novas equipes 72; e, já no contexto da pandemia, a instituição da Agência para o Desenvolvimento da Atenção Primária à Saúde 73, na forma de pessoa jurídica de direito privado sem fins lucrativos, que tem sido apontada como tendo natureza jurídica semelhante à de uma organização social 74 .

A ausência de ações com foco no monitoramento da realização do DHAA ficou clara. Os documentos usados como referencial teórico 14,15 apontam a importância de os governos coletarem e compartilharem dados, bem como apoiarem pesquisas sobre o impacto nos sistemas alimentares, na garantia da segurança alimentar e nutricional e sobre as medidas tomadas para conter os impactos sobre o DHAA. Os retrocessos institucionais e orçamentários na agenda da segurança alimentar e nutricional 75,76 já sinalizados, ocorridos no período pré-pandemia e não revertidos durante a crise sanitária atual, também foram expressados na extinção do Conselho Nacional de Segurança Alimentar e Nutricional (CONSEA), como um dos primeiros atos do Governo Federal, órgão cuja missão era propor diretrizes para assegurar o DHAA 77; inoperância da Câmara Interministerial de Segurança Alimentar e Nutricional (CAISAN); não realização da 6a Conferência Nacional de Segurança Alimentar e Nutricional e a ausência do III Plano Nacional de Segurança Alimentar e Nutricional (PLANSAN), que pauta o planejamento e a execução da PNSAN. Tais medidas comprometeram o monitoramento às violações do DHAA no contexto da pandemia pelo Governo Federal, cujos arranjos institucionais propostos até então não dão conta ou não têm como atribuição o monitoramento do DHAA. Ainda no que diz respeito à garantia e monitoramento do DHAA, vale ressaltar a publicação da Portaria no 683 78, de 19 de março de 2020, que dispõe sobre a instituição de um comitê técnico para a elaboração de iniciativas de promoção e defesa dos Direitos Humanos durante a pandemia. No entanto, nenhum arranjo institucional presente na Portaria é responsável diretamente pelo DHAA. 
O cenário de retrocessos, que envolvem congelamento dos gastos sociais, reformas da previdência social e da legislação trabalhista, vai afetar o acesso aos alimentos pelos mais pobres, recolocando o país no Mapa da Fome 79. Essa situação - atrelada à pandemia atual - irá tornar ainda mais desafiador o cumprimento da Agenda 2030. Não foi identificada nenhuma menção à revogação da Emenda Constitucional no 95 80, chamada de "teto dos gastos", que afeta as despesas primárias. Essa medida é apontada pelo documento do FBSSAN 10 como uma ação estratégica para enfrentar as consequências da pandemia.

Cabe destacar como limitações deste artigo a dificuldade em acessar informações detalhadas acerca das ações disponíveis nos próprios boletins e homepages de ministérios setoriais, visto que as mesmas eram apresentadas de forma muito sintéticas, o que pode limitar a análise de suas potencialidades e dificuldades.

\section{Considerações finais}

Levando-se em conta a capacidade de resposta do Governo Federal no contexto da COVID-19 e os retrocessos nos últimos anos, cabe tecer algumas breves reflexões. A primeira delas diz respeito à capacidade de respostas emergenciais frente à pandemia pelo que restou do SISAN, após tantos desmontes, esvaziamentos e desarticulações institucionais, além de reduções orçamentárias. A liberação de créditos extraordinários seria capaz de reverter esse cenário? Outra questão é: frente à extinção do CONSEA e à inoperância da CAISAN, o desafio de pautar ações e monitorar os impactos da pandemia na segurança alimentar e nutricional, de maneira articulada, está posto. Outro desafio reside na participação da sociedade civil no processo de planejamento e monitoramento da realização do DHAA que, atualmente, se restringe à possibilidade de doações financeiras no âmbito do Governo Federal. Não pretende-se aqui esgotar a discussão sobre os retrocessos, mas destacar que a perspectiva intersetorial e o envolvimento dos diferentes atores institucionais e da sociedade civil, aspectos tão centrais na consolidação do SISAN, foram intencionalmente desarticulados.

As ações do Governo Federal para a mitigação dos efeitos da COVID-19 até então propostas residem em medidas emergenciais que focam principalmente no acesso à renda e aos alimentos. Entretanto, a garantia do DHAA e a concretização da segurança alimentar e nutricional exigem, além da articulação intersetorial, ações coordenadas não apenas emergenciais que busquem atenuar efeitos de crises, mas medidas a médio e longo prazos que possam garantir o direito constitucional à alimentação. A sensação de insegurança da população frente às incertezas no contexto da pandemia é amplificada pela crise política que se instala neste momento, além das orientações contraditórias do Poder Público.

É no mínimo curioso notar que o discurso atual contra o isolamento social em função dos possíveis efeitos de aumento da fome no país emerge de um governo que, há meses atrás, negou a existência da mesma. A agenda de segurança alimentar e nutricional foi se fortalecendo no país nos últimos 15 anos, e nos três últimos seu desmonte vem acontecendo com a extinção do CONSEA e outros retrocessos, por exemplo, nas políticas de incentivo à agricultura familiar, de acesso à água e de abastecimento alimentar, afetando diretamente a concretização da segurança alimentar e nutricional e do DHAA.

Por fim, este momento da pandemia coloca em foco o maior problema do Brasil, as desigualdades sociais. Essas, por sua vez, exacerbam os efeitos da COVID-19 nas condições de vida da população. Dessa maneira, é necessário pensar, discutir e formular políticas públicas nacionais que tenham como base a economia e a proteção social, mas que estejam articuladas com as diretrizes da PNSAN na perspectiva da garantia do DHAA. 


\section{Colaboradores}

T. M. A. Alpino, C. R. B. Santos e D. C. Barros participaram da concepção e planejamento do estudo, levantamento e análise dos dados, elaboração do texto, revisão e aprovação da versão final do manuscrito. C. M. Freitas participou da concepção do estudo, análise dos dados, elaboração do texto, revisão e aprovação da versão final do manuscrito.

\section{Informações adicionais}

ORCID: Tais de Moura Ariza Alpino (0000-00015045-9483); Cláudia Roberta Bocca Santos (00000002-4312-3049); Denise Cavalcante de Barros (0000-0001-5016-0844); Carlos Machado de Freitas (0000-0001-6626-9908).

\section{Referências}

1. Freitas ARR, Napimoga M, Donalisio MR. Análise da gravidade da pandemia de Covid-19. Epidemiol Serv Saúde 2020; 29:e2020119.

2. Barreto ML, Barros AJDD, Carvalho MS, Codeço CT, Hallal PRC, Medronho RDA, et al. $\mathrm{O}$ que é urgente e necessário para subsidiar as políticas de enfrentamento da pandemia de COVID-19 no Brasil? Rev Bras Epidemiol 2020; 23:e200032.

3. Arrais TPA, Oliveira ARD, Alencar D, Salgado TR, Lima LDO, Viana JLR, et al. Relatório: Pandemia COVID-19: o caráter emergencial das transferências de renda direta e indireta para a população vulnerável do Estado de Goiás. Espaço e Economia: Revista Brasileira de Geografia Econômica 2020; IX:13734.

4. Valfré V. Bolsonaro volta a criticar isolamento social: não dá para continuar assim. Estadão 2020; 26 mai. https://noticias.uol.com.br/ul timas-noticias/agencia-estado/2020/05/26/ bolsonaro-volta-a-criticar-isolamento-socialnao-da-para-continuar-assim.htm.

5. Bezerra ACV, Silva, CEM, Soares FRG, Silva JAM. Fatores associados ao comportamento da população durante o isolamento social na pandemia de COVID-19. Ciênc Saúde Colet 2020; 25 Suppl 1:2411-21

6. Oliveira M, Mello I. Subnotificação, crise, polarização: razões para baixa adesão ao isolamento. UOL 2020; 18 abr. https://noticias. uol.com.br/saude/ultimas-noticias/reda cao/2020/04/18/subnotificacao-faz-popula cao-relaxar-e-aumentar-proliferacao-dacovid-19.htm.

7. Moraes RFD. COVID-19 e medidas legais de distanciamento social: tipologia de políticas estaduais e análise do período de 13 a 26 de abril de 2020. Brasília: Instituto de Pesquisa Econômica Aplicada; 2020. (Nota Técnica, 18).

8. Troca de ministros afeta todo o sistema de saúde, afirma secretário de SP. UOL 2020; 19 mai. https://noticias.uol.com.br/saude/ultimas-no ticias/redacao/2020/05/19/troca-de-minis tros-afeta-todo-o-sistema-de-saude-afirmasecretario-de-sp.htm.

9. Mello G, Oliveira ALM, Guidolin AP, Caso C, David G, Nascimento JC, et al. A coronacrise: natureza, impactos e medidas de enfrentamento no Brasil e no mundo. https://www. eco.unicamp.br/images/arquivos/nota_ce con_coronacrise_natureza_impactos_e_me didas_de_enfrentamento.pdf (acessado em 22/ Abr/2020).

10. Fórum Brasileiro de Soberania e Segurança Alimentar e Nutricional. Garantir o direito à alimentação e combater a fome em tempos de coronavírus. Aliança pela Alimentação Adequada e Saudável. https://alimentacaosauda vel.org.br/garantir-o-direito-a-alimentacao-ecombater-a-fome-em-tempos-de-coronavi rus/6243/ (acessado em 28/Abr/2020). 
11. Global Network Against Food Crises; Food Security Information Network. Global Report on Food Crises. Joint analisys for better decisions. Washington DC: International Food Policy Research Institute; 2020.

12. Brasil está voltando ao mapa da fome, diz diretor da ONU. Exame 2020; 12 mai. https://exa me.com/brasil/brasil-esta-voltando-ao-mapada-fome-diz-diretor-da-onu/.

13. Cullen MT. Coronavirus food supply chain under strain. What to do? Food systems transformation. Roma: Food and Agriculture Organization of the United Nations; 2020.

14. FIAN Internacional. Impact of COVID-19 on the Human Right to Food and Nutrition. Preliminary monitoring report. https://hilalelver. org/category/food-in-the-news/covid-19/ (acessado em 28/Abr/2020).

15. Committee on World Food Security. Impact of COVID-19 on Food Security and Nutrition (FSN). Roma: Food and Agriculture Organization of the United Nations; 2020.

16. Rother ET. Revisão sistemática $X$ revisão narrativa. Acta Paul Enferm 2007; 20:v-vi.

17. Maluf R. Comer em tempos de pandemia e após. Jornal GGN 2020; 3 abr. https://jor nalggn.com.br/a-grande-crise/comer-emtempos-de-pandemia-e-apos-por-renato-smaluf/.

18. The International Panel of Experts on Sustainable Food Systems. COVID-19 and the crisis in food systems: symptoms, causes, and potential solutions. Bruxelas: The International Panel of Experts on Sustainable Food Systems; 2020.

19. Global Food Security Cluster. Coronavirus, impact on well-being, health, food access and food security. https://fscluster.org/sites/de fault/files/documents/covid_19_impact_on_ food_security.pdf (acessado em 14/Mai/2020).

20. Marchisio M. The potential impact of COVID-19 on SDG 2 (food security) - in China and globally. International Fund for Agricultural Development. https://www.ifad.org/ en/web/latest/blog/asset/41828816 (acessado em 13/Mar/2020).

21. Brasil. Decreto no 10.211 , de 30 de janeiro de 2020. Dispõe sobre o Grupo Executivo Interministerial de Emergência em Saúde Pública de Importância Nacional e Internacional GEI-ESPII. Diário Oficial da União 2020; 30 jan.

22. Brasil. Decreto no 10.238 , de 11 de fevereiro de 2020. Altera o Decreto no 10.211 , de 30 de janeiro de 2020, que dispõe sobre o Grupo Executivo Interministerial de Emergência em Saúde Pública de Importância Nacional e Internacional - GEI-ESPII. Diário Oficial da União 2020; 12 fev.

23. Ministério da Saúde. Portaria no 188 , de 3 de fevereiro de 2020. Declara Emergência em Saúde Pública de importância Nacional (ESPIN) em decorrência da Infecção Humana pelo novo Coronavírus (2019-nCoV). Diário Oficial da União 2020; 4 fev.
24. Brasil. Decreto no 10.277 , de 16 de março de 2020. Institui o Comitê de Crise para Supervisão e Monitoramento dos Impactos da Covid-19. Diário Oficial da União 2020; 16 mar.

25. Brasil. Lei no 13.982, de 2 de abril de 2020. Altera a Lei no 8.742, de 7 de dezembro de 1993 , para dispor sobre parâmetros adicionais de caracterização da situação de vulnerabilidade social para fins de elegibilidade ao Benefício de Prestação Continuada (BPC), e estabelece medidas excepcionais de proteção social a serem adotadas durante o período de enfrentamento da Emergência de Saúde Pública de Importância Internacional decorrente do Coronavírus (Covid-19) responsável pelo surto de 2019, a que se refere a Lei no 13.979 , de 6 de fevereiro de 2020. Diário Oficial da União 2020; 2 abr.

26. Sampaio C. Câmara aprova auxílio de R $\$ 600$ que pode beneficiar 24 milhões de informais. Brasil de Fato 2020; 26 mar. https://www.bra sildefato.com.br/2020/03/26/camara-apro va-auxilio-de-r-600-para-trabalhadores-infor mais-durante-crise.

27. Brasil. Decreto no 10.412 , de 30 de junho de 2020. Altera o Decreto no 10.316 , de 7 de abril de 2020, para prorrogar o período de pagamento do auxílio emergencial de que trata a Lei no 13.982, de 2 de abril de 2020. Diário Oficial da União 2020; 1 jul.

28. Tomazelli I, Fernandes A. Transferência de renda é adotada em 30 países. UOL Economia 2020; 24 mar. https://economia.uol.com. $\mathrm{br} /$ noticias/estadao-conteudo/2020/03/24/ transferencia-de-renda-e-adotada-em-30-pai ses.htm.

29. Câmara dos Deputados. Projeto de Lei no 873 , de 2020. https://www25.senado.leg.br/web/ atividade/materias/-/materia/141614 (acessado em 08/Abr/2020).

30. Bolsonaro sanciona com vetos ampliação do auxílio emergencial de R\$600. UOL Economia 2020; 15 mai. https://economia.uol.com.br/ noticias/reuters/2020/05/15/bolsonaro-san ciona-com-vetos-ampliacao-do-auxilio-emer gencial-de-r600.htm.

31. Presidência da República. Despachos do Presidente da República. Diário Oficial da União 2020; 15 mai.

32. Menezes F, Burity V. O novo veto do Capitão da Fome. Le Monde Diplomatique Brasil 2020; 22 mai. https://diplomatique.org.br/o-novoveto-do-capitao-fome/.

33. Pichonelli M. Por que você não deve falar "coronavoucher" e estigmatizar mais as pessoas. UOL 2020; 3 abr. https://www.uol.com.br/ ecoa/ultimas-noticias/2020/04/03/por-quevoce-nao-deve-falar-coronavoucher-e-estig matizar-mais-as-pessoas.htm.

34. Marchesan R. Centralizar auxílio de R\$ 600 na Caixa criou fila e atraso, dizem analistas. UOL Economia 2020; 6 mai. https://economia.uol. com.br/noticias/redacao/2020/05/06/auxi lio-emergencial-problemas-pagamento-caixa. htm. 
35. Agência Brasil. Cerca de 20 milhões de pessoas sem conta já sacaram o auxílio emergencial. UOL Economia 2020; 8 mai. https://economia. uol.com.br/noticias/redacao/2020/05/08/cerca-de-20-milhoes-de-pessoas-sem-conta-jasacaram-o-auxilio-emergencial.htm.

36. Ministério da Cidadania. Portaria no 387, de 15 de maio de 2020. Altera a Portaria no 335, de 20 de março de 2020, para acrescentar medidas emergenciais na gestão do Programa Bolsa Família, criado pela Lei no 10.836, de 9 de janeiro de 2004, e do Cadastro Único para Programas Sociais do Governo Federal, regulamentado pelo Decreto no 6.135, de 26 de junho de 2007, em decorrência da Emergência em Saúde Pública de Importância Nacional. Diário Oficial da União 2020; 18 mai.

37. Burlandy L. Transferência condicionada de renda e segurança alimentar e nutricional. Ciênc Saúde Colet 2007; 12:1441-51.

38. Rasella D, Aquino R, Santos CAT, Sousa Paes R, Barreto ML. Effect of a conditional cash transfer programme on childhood mortality: a nationwide analysis of Brazilian municipalities. Lancet 2013; 382:57-64.

39. Martins AMBB. Avaliação dos impactos de políticas públicas de transferência de renda na qualidade de vida no semiárido nordestino face às mudanças climáticas [Tese de Doutorado]. São Paulo: Faculdade de Saúde Pública, Universidade de São Paulo; 2016.

40. Santarelli M, David G, Burity V, Rocha NZ. Informe Dhana 2019: autoritarismo, negação de direitos e fome. Brasília: FIAN Brasil; 2019.

41. Santarelli M, Burity V, Silva LNB, Prates L, Rizzolo A, Rocha NC. Da democratização ao golpe: avanços e retrocessos na garantia do direito humano à alimentação e à nutrição adequadas no Brasil. Brasília: FIAN Brasil; 2017.

42. Melito L. Arquitetura da destruição das políticas de combate à fome no Brasil. O Joio e o Trigo 2020; 3 fev. https://outraspalavras.net/ ojoioeotrigo/2020/02/arquitetura-da-des truicao-das-politicas-de-combate-a-fome-nobrasil/.

43. Brasil. Lei no 13.987, de 7 de abril de 2020. Altera a Lei no 11.947 , de 16 de junho de 2009 , para autorizar, em caráter excepcional, durante o período de suspensão das aulas em razão de situação de emergência ou calamidade pública, a distribuição de gêneros alimentícios adquiridos com recursos do Programa Nacional de Alimentação Escolar (PNAE) aos pais ou responsáveis dos estudantes das escolas públicas de educação básica. Diário Oficial da União 2020; 7 abr.

44. Ministério da Agricultura, Pecuária e Abastecimento; Ministério da Educação. Orientações para a execução do PNAE durante a situação de emergência decorrente da pandemia do Coronavírus (covid-19). Brasília: Ministério da Agricultura, Pecuária e Abastecimento/Ministério da Educação; 2020.
45. Fórum Brasileiro de Soberania e Segurança Alimentar e Nutricional. Alimentação escolar: defender o direito à alimentação em tempos de pandemia. Facebook Watch. https://www.facebook.com/watch/live/? $\mathrm{v}=613355662605041 \&$ ref $=$ watch_permalink (acessado em 22/Mai/2020).

46. Tribunal de Justiça do Estado do Rio de Janeiro. Ação Civil Pública. Processo 009347252.2020.8.19.0001. http://www.mprj.mp.br/ documents/20184/540394/deciso_acp_ali mentao_escolar__rio_de_janeiro__proibio_ da_abertura_de_escolas.pdf (acessado em 01/ $\mathrm{Jul} / 2020$ ).

47. Valadares AA, Alves F, Galiza M, Silva SP. Agricultura familiar e abastecimento alimentar no contexto do Covid-19: uma abordagem das ações públicas emergenciais. Brasília: Instituto de Pesquisa Econômica Aplicada; 2020. (Nota Técnica, 69).

48. Ministério da Cidadania. Portaria no 369, de 29 de abril de 2020. Dispõe acerca do atendimento do Cadastro Único para Programas Sociais do Governo Federal - Cadastro Único, disposto pelo Decreto no 6.135, de 26 de junho de 2007, no Distrito Federal e nos municípios que estejam em estado de calamidade pública ou em situação de emergência reconhecidos pelos governos estadual, municipal, do Distrito Federal ou Federal, inclusive a Emergência de Saúde Pública de Importância Internacional declarada pela Organização Mundial da Saúde, em 30 de janeiro de 2020, em decorrência da Infecção Humana pelo novo Coronavírus (COVID-19). Diário Oficial da União 2020; 30 abr.

49. Ministério da Cidadania. Portaria no 385 , de 13 de maio de 2020. Dispõe sobre repasse emergencial de recursos federais para a operacionalização de ações de incremento à segurança alimentar e nutricional aos usuários do Sistema Único de Assistência Social - SUAS, no âmbito da União, dos estados, Distrito Federal e municípios devido à situação de Emergência em Saúde Pública de Nacional - ESPIN, em decorrência de infecção humana pelo novo Coronavírus, Covid-19. Diário Oficial da União 2020; 14 mai.

50. Brasil. Medida Provisória no 924, de 13 de março de 2020. Abre crédito extraordinário, em favor dos Ministérios da Educação e da Saúde, no valor de R\$ 5.099.795.979,00, para os fins que especifica. Diário Oficial da União 2020; 13 mar.

51. Brasil. Medida Provisória no 940, de 2 de abril de 2020. Abre crédito extraordinário, em favor do Ministério da Saúde, no valor de R\$ 9.444.373.172,00, para os fins que especifica. Diário Oficial da União 2020; 2 abr. 
52. Brasil. Medida Provisória no 941, de 2 de abril de 2020. Abre crédito extraordinário, em favor dos Ministérios da Educação, da Saúde e da Cidadania, no valor de R\$ 2.113.789.466,00, para os fins que especifica. Diário Oficial da União 2020; 2 abr.

53. Brasil. Medida Provisória no 957, de 24 de abril de 2020. Abre crédito extraordinário, em favor do Ministério da Cidadania, no valor de R\$ 500.000.000,00, para o fim que especifica. Diário Oficial da União 2020; 27 abr.

54. Secretaria Especial do Desenvolvimento Social, Ministério da Cidadania. Programa de aquisição de alimentos recebe crédito extra de R\$ 500 milhões. http://desenvolvimentosocial. gov.br/imprensa/Noticias/programa-de-aqui sicao-de-alimentos-recebe-credito-extra-der-500-milhoes (acessado em 28/Abr/2020).

55. Geitens JF. Após pressão por MP, sociedade civil cobra execução de programa alimentar durante a pandemia. O Joio e o Trigo 2020; 30 abr. https://outraspalavras.net/ojoioeotri go/2020/04/apos-pressao-por-medida-provi soria-sociedade-civil-cobra-execucao-de-pro grama-de-alimentos-durante-a-pandemia/.

56. Matioli V, Peres J. Coronavírus: Brasil não tem estoque de alimentos para enfrentar desabastecimento. O Joio e o Trigo 2020; 26 mar. https://outraspalavras.net/ojoioeotrigo/ 2020/03/coronavirus-brasil-nao-tem-esto que-de-alimentos-para-enfrentar-desabasteci mento/.

57. Banco Central do Brasil. Resolução no 4.810, de 30 de abril de 2020. Estabelece medidas de caráter emergencial para os procedimentos relativos à concessão, ao controle e à fiscalização das operações de crédito rural em decorrência das medidas de distanciamento social adotadas para mitigar os impactos da pandemia provocada pela Covid-19. Diário Oficial da União 2020; 5 mai.

58. Sardinha E. Agronegócio ignora crise e cresce mesmo com a pandemia de covid-19. Congresso em Foco 2020; 28 abr. https://congres soemfoco.uol.com.br/economia/agronego cio-ignora-crise-e-cresce-mesmo-com-pande mia-de-covid-19/.

59. Ministério da Agricultura, Pecuária e Abastecimento. Instrução Normativa no 29, de 23 de abril de 2020. Estabelece os requisitos para o livre comércio de produtos de origem animal, inspecionados por consórcio público de Municípios. Diário Oficial da União 2020; 24 abr.

60. Sudré L. Mesmo com pandemia, governo Bolsonaro já liberou 150 novos agrotóxicos este ano. Brasil de Fato 2020; 13 mai. https://www. brasildefato.com.br/2020/05/13/mesmo-com -pandemia-governo-bolsonaro-ja-liberou150-novos-agrotoxicos-este-ano.

61. Sudré L. Liberação de agrotóxicos no governo Bolsonaro é a maior dos últimos 14 anos. Brasil de Fato 2019; 27 nov. https://www.bra sildefato.com.br/2019/11/27/liberacao-de-a grotoxicos-no-governo-bolsonaro-e-a-maiordos-ultimos-14-anos.
62. Oliveira L, Tooge R. Número de agrotóxicos registrados em 2019 é o maior da série histórica; 94,5\% são genéricos, diz governo. G1 2019; 28 dez. https://g1.globo.com/economia/agro negocios/noticia/2019/12/28/numero-de-a grotoxicos-registrados-em-2019-e-o-maiorda-serie-historica-945 percent-sao-genericosdiz-governo.ghtml.

63. Secretaria de Política Agrícola, Ministério da Agricultura, Pecuária e Abastecimento. Portaria no 11 , de 6 de abril de 2020. Diário Oficial da União 2020; 8 abr.

64. Silva NGA, Souza JPOD. Seguro Safra para o semi-árido cearense. In: 46o Congresso Sociedade Brasileira de Economia, Administração e Sociologia Rural. https://ageconsearch. umn.edu/record/133290/ (acessado em 26/ Mai/2020).

65. Secretaria de Vigilância em Saúde; Secretaria de Atenção Primária à Saúde, Ministério da Saúde. Recomendações de alimentação e COVID-19. Brasília: Ministério da Saúde; 2020.

66. Departamento de Atenção Básica, Secretaria de Atenção Básica, Ministério da Saúde. Guia alimentar para a população brasileira. Brasília: Ministério da Saúde; 2014

67. Faculdade de Saúde Pública, Universidade de São Paulo. 8a Webinar: Insegurança Alimentar e Nutricional em tempos de Covid-19. Vídeo; 2:20min. https://www.youtube.com/watch? $\mathrm{v}=\mathrm{PhZejewNlz} 4$.

68. Empresa Brasileira de Pesquisa Agropecuária. Consumo de hortaliças na quarentena. https:// www.embrapa.br/hortalicas/pesquisa-consu mo-covid19 (acessado em 30/Jun/2020).

69. Santos TA. Dilemas políticos para o semiárido brasileiro: um breve panorama até a crise do Lulismo. NERA: Revista Núcleo de Estudos, Pesquisas e Projetos de Reforma Agrária 2019; 22:170-94.

70. Coordenação Geral de Alimentação e Nutrição, Departamento de Promoção da Saúde, Secretaria de Atenção Primária à Saúde, Ministério da Saúde. Ofício Circular no 3/2020/ CGAN/DEPROS/SAPS/MS. Solicitação de apoio logíscotico para execução da pesquisa intulada Evolução da Prevalência de Infecção por Covid-19 no Brasil: estudo de base populacional. Brasília: Ministério da Saúde; 2020.

71. Ministério da Saúde. Portaria no 2.979, de 12 de novembro de 2019. Institui o Programa Previne Brasil, que estabelece novo modelo de financiamento de custeio da Atenção Primária à Saúde no âmbito do Sistema Único de Saúde, por meio da alteração da Portaria de Consolidação no 6/GM/MS, de 28 de setembro de 2017. Diário Oficial da União 2019; 13 nov.

72. Departamento de Saúde da Família, Secretaria de Atenção Primária à Saúde, Ministério da Saúde. Nota Técnica no 3/2020-DESF/SAPS/ MS. Núcleo Ampliado de Saúde da Família e Atenção Básica (NASF-AB) e Programa Previne Brasil. Brasília: Ministério da Saúde; 2020. 
73. Brasil. Decreto no 10.283 , de 20 de março de 2020. Institui o Serviço Social Autônomo denominado Agência para o Desenvolvimento da Atenção Primária à Saúde - Adaps. Diário Oficial da União 2020; 20 mar.

74. Associação Brasileira de Nutrição. Criação da ADAPS: ataque ao SUS em meio à crise do Coronavírus. https://asbran.org.br/noticias/ criacao-da-adaps-ataque-ao-sus-em-meioa-crise-do-coronavirus (acessado em 23/ Mar/2020).

75. Câmara Interministerial de Segurança Alimentar e Nutricional. Plano Nacional de Segurança Alimentar e Nutricional: 2012/2015. Brasília: Câmara Interministerial de Segurança Alimentar e Nutricional; 2011.

76. Câmara Interministerial de Segurança Alimentar e Nutricional. Plano Nacional de Segurança Alimentar e Nutricional (PLANSAN-2016-2019). Brasília: Ministério do Desenvolvimento Social e Agrário; 2017.

77. Castro IRR. A extinção do Conselho Nacional de Segurança Alimentar e Nutricional e a agenda de alimentação e nutrição. Cad Saúde Pública 2019; 35:e00009919.

78. Ministério da Mulher, da Família e dos Direitos Humanos. Portaria no 683, de 19 de março de 2020. Dispõe sobre a instituição de comitê técnico para elaboração de iniciativas de promoção e defesa dos Direitos Humanos, considerando a situação de emergência de saúde pública de importância internacional decorrente do Coronavírus (COVID-19). Diário Oficial da União 2020; 20 mar.

79. Grupo de Trabalho da Sociedade Civil para Agenda 2030. Relatório luz da Agenda 2030 de desenvolvimento sustentável: síntese. http://actionaid.org.br/wp-content/files_ mf/1499785232Relatorio_sintese_v2_23jun. pdf (acessado em 02/Mai/2020).

80. Brasil. Emenda Constitucional no 95, de 15 de dezembro de 2016. Altera o Ato das Disposições Constitucionais Transitórias, para instituir o Novo Regime Fiscal, e dá outras providências. Diário Oficial da União 2016; 15 dez.

81. Ministério da Agricultura, Pecuária e Abastecimento. Portaria no 123 , de 30 de março de 2020. Institui, no âmbito do Ministério da Agricultura, Pecuária e Abastecimento MAPA, o Comitê de Crise (CC-AGROCOVID19). Diário Oficial da União 2020; $31 \mathrm{mar}$.

82. Brasil. Decreto no 10.311 , de 3 de abril de 2020. Institui o Conselho de Solidariedade para Combate à Covid-19 e aos seus Efeitos Sociais e Econômicos. Diário Oficial da União 2020; 3 abr.

83. Ministério da Agricultura, Pecuária e Abastecimento. Portaria no 116 , de 26 de março de 2020. Dispõe sobre os serviços, as atividades e os produtos considerados essenciais pelo $\mathrm{Mi}$ nistério da Agricultura, Pecuária e Abastecimento para o pleno funcionamento das cadeias produtivas de alimentos e bebidas, para assegurar o abastecimento e a segurança alimentar da população brasileira enquanto perdurar o estado de calamidade pública decorrente da pandemia da COVID-19. Diário Oficial de União 2020; 27 mar.

84. Ministério da Agricultura, Pecuária e Abastecimento. Portaria no 24, de 24 de março de 2020. Prorroga o prazo de validade da Declaração de Aptidão ao Pronaf (DAP), na calamidade pública, reconhecida pelo Decreto Legislativo no 6, de 20 de março de 2020, do Congresso Nacional, decorrente da pandemia causada pelo vírus Covid-19. Diário Ofical da União 2020; 25 mar.

85. Brasil. Decreto no 6.040, de 7 de fevereiro de 2007. Institui a Política Nacional de Desenvolvimento Sustentável dos Povos e Comunidades Tradicionais. Diário Oficial da União 2007; 8 fev.

86. Brasil. Lei no 11.346 , de 15 de setembro de 2006. Cria o Sistema Nacional de Segurança Alimentar e Nutricional - SISAN com vistas em assegurar o direito humano à alimentação adequada e dá outras providências. Diário Oficial da União 2006; 18 set. 


\section{Abstract}

The COVID-19 pandemic poses one of this century's greatest public health challenges, with impacts on the health and living conditions of populations worldwide. The literature has reported that the pandemic affects the hegemonic food system in various ways. In Brazil, the pandemic amplifies existing social, racial, and gender inequalities, further jeopardizing the Human Right to Adequate Food (HRAF) and the attainment of food and nutritional security, especially among more vulnerable groups. In this context, the article aims to analyze the first measures by the Brazilian Federal Government to mitigate the pandemic's effects and that may have repercussions on food and nutritional security, considering the recent institutional changes in policies and programs. A narrative literature review was performed, and the information sources were the bulletins of the Center for Coordination of Operations by the Crisis Committee for Supervising and Monitoring the Impacts of COVID-19 and homepages of various government ministries, from March to May 2020. The actions were systematized according to the guidelines of the National Policy for Food and Nutritional Security. The analysis identified the creation of institutional crisis management arrangements. The proposed actions feature those involving access to income, emergency aid, and food, such as authorization for food distribution outside schools with federal funds from the $\mathrm{Na}$ tional School Feeding Program. However, the setbacks and dismantlement in food and nutritional security may undermine the Federal Government's capacity to respond to COVID-19.

Food and Nutrition Security; COVID-19; Public Policy

\section{Resumen}

La pandemia por COVID-19 representa uno de los mayores desafíos de la salud pública de este siglo, causando impactos en la salud y condiciones de vida de las poblaciones en todo el mundo. Se ha señalado por parte de la literatura que la pandemia afecta de diversas formas el sistema alimentario hegemónico. En Brasil, la pandemia amplifica las desigualdades sociales, raciales y de género ya existentes, comprometiendo todavía más la garantía del Derecho Humano a la Alimentación Adecuada (DHAA) y la concretización de la seguridad alimentaria y nutricional, especialmente entre los más vulnerables. En este contexto, el objetivo de este artículo es analizar las primeras acciones, en el ámbito federal, del gobierno brasileño para la mitigación de los efectos de la pandemia que puedan repercutir en la seguridad alimentaria y nutricional, considerando los recientes cambios institucionales de las politicas y programas. Se realizó una revisión narrativa de la literatura y se utilizaron como fuentes de información los boletines del Centro de Coordinación de Operaciones del Comité de Crisis para la Supervisión y Monitoreo de los Impactos de la COVID-19y homepages de ministerios sectoriales, de marzo a mayo de 2020. Las acciones se sistematizaron según las directrices de la Politica Nacional de Seguridad Alimentaria y Nutricional. Se identificó la creación de soluciones institucionales para la gestión de la crisis. Entre las acciones propuestas, se destacan aquellas relacionadas con el acceso a la renta, como el apoyo de emergencia, $y$ de alimentos, como la autorización para la distribución de alimentos fuera del ambiente escolar con los recursos federales del Programa Nacional de Alimentación Escolar. No obstante, los retrocesos y recortes en el área de seguridad alimentaria y nutricional pueden comprometer la capacidad de respuesta del Gobierno Federal en el contexto de la COVID-19.

Seguridad Alimentaria y Nutricional; COVID-19; Política Pública
Recebido em 09/Jun/2020

Versão final reapresentada em 02/Jul/2020

Aprovado em 07/Jul/2020 